\title{
Smart Veggie Identification and Alerting System for Supermarkets using Image Processing Techniques and Neural Networks
}

\author{
Hewasinghe H. H. K \\ Faculty of Computing \\ General Sir John Kotelawala Defence University \\ Rathmalana, Sri Lanka
}

\author{
Pemarathne W. P. J \\ Faculty of Computing \\ General Sir John Kotelawala Defence University \\ Rathmalana, Sri Lanka
}

\begin{abstract}
The recent advances in the field of image processing has become a powerful quantitative method in development of science and engineering. Image recognition is one of the foremost areas in computer vision, it yields high degree understanding through computers, one of the maximum crucial regions in recognition is object recognition that's the process of locating a specific object in an image or video surveillance. This paper presents the current state of image processing techniques to identify vegetables and fruits and its capabilities to improve hardware resources with the growing demands of the business industry. The proposed system can identify vegetables and fruits in a basket that are to be retailed and alert the authorities when a basket is going to be finished. The application is based on color and size comparison of the vegetables and fruits in a live video with a reference image and there by extract similar features by using neural network for identification. Baskets are marked with a colored line to indicate the level of the item. Particular level is identified through color identification and notify the responsible parties.
\end{abstract}

\section{Keywords}

Image processing, Object recognition, neural network, Color identification, Fruits and vegetable recognition

\section{INTRODUCTION}

IT for business alignment is a dynamic state in which a business organization can use information technology (IT) effectively to achieve business objectives and improve financial performance or marketplace competitiveness. With the development of technology many revolutions have been occurred in business-to- business, business to consumer or consumer to consumer transactions. The traditional grocery store idea has been evolving in to a "mega shops" and the heavy discounter idea which carry all the goods shoppers need. If we observe the drivers for the evolution of current supply chains, or "contemporary trade" they had been chronic in boom demand for cost added client products, comfort food, beverage, and frozen confectioneries [1], [2]. Usually customers have an idea what they want to buy. This is most because of today's customers browse internet daily and other TV commercials aware them about the best products to pick. For an example, new analysis reveals that the "experiential factor" is driving today's foodstuff shoppers who are seeking positive preparation experiences, creating deliberate selections from the shop to the stove, as well as needing to feel smart regarding the food they eat. They have pride within the brands they buy and share their change of state journeys on-line [3], [4].
But after doing a research it was concluded that the sellers should much more aware about the customer preferences to increase their sales. A system that tracks the images of the items displayed and triggers an alarm when it is going to be finished to inform the responsible parties that an item is finished, and refill is identified as the best solution. So, that they can avoid customer disappointment as well as they can get an idea about the daily demand of that item. Since the system can identify the customer preferences, they know the demand and can improve sales from that item. This proposed system will revolutionize` the modern supermarket culture.

\section{LITERATURE REVIEW \\ 2.1 Background}

According to the current context in Sri Lanka and other countries, there exists lot of management systems in use. As per the conducted research most of them are college/university management systems and it is very rare to find an automatic management systems in supermarkets especially in Sri Lanka. In developed countries like England supermarkets use developed technologies for their management. For an example "TESCO" chain has the super management system for their inventory control, identify customer behavior and effective way of billing process. They are tricky enough to keep their loyal customers in touch. One of the best examples is the "Broccoli Cam" they have introduced to detect empty broccoli baskets.

Lu, Kiewit and Zhang [5] invented an automated system and a method which collects market research data with retail customer data together with retail sales data. He has fixed cameras on racks to observe customer behaviors. This framework and strategy fit of recognizing a retail client with sufficient precision to have the capacity to gauge the shopping recurrence at guaranteed store, and that defeats many the burdens of earlier workmanship techniques for recognizing successive customers in a retail location. In this system Eigenface recognition is used for face recognition.

Ali and Sonkusare [6] in their paper present the RFID based smart shopping and billing system. The system consists of Cart location detection unit (CLDU), Server Communication unit (SCU), User Interface and display unit (UIDU) and Billing and Inventory management unit (BIMU). At the point when a client with the cart enters a shopping passageway, the cart is tracked in scope of the IR receiver and the microcontroller checks for the path data code. The walkway data code is transmitted over the ZigBee remote from the cart to the server. Based on the path number got the database is questioned and significant data is recovered and transmitted to the cart by means of the ZigBee module. The got data is put away in the EEPROM display on the cart. This fills in as 
a transitory database until the client leaves the specific path that he/she is in. The important items data is shown on the show unit. Each item has a RFID label which contains a Unique ID. These ID ${ }^{\text {ees }}$ is nourished in the database relegated to the relating items. If there should be a buy done, then that item can be dropped in the cart where the RFID reader reads the tag. The data of the item is extricated and shown on the LCD screen.

In the meantime, billing data is updated. The exit information is sent to the server alongside subtle elements of purchase. Server then stores them in the database. These means are rehashed until the finish of shopping round is finished. Once the "Entire" round is finished there is a choice gave to end the shopping with similar items or to remove the items from the cart. This passes by the client choice. At the finish of shopping, the client can straight away pay the bill and leave. Inventory status of the items is likewise updated at the end of shopping. They propose to develop the system with more secured communication and increase the efficiency of the time by integrating with another ZigBee module. Further they also propose to add sophisticated microcontroller and a large display unit.

Sojitra and Patel [1] proposed a system to avoid the long waiting queue at the billing counter. They concluded to design a system with NFC tags and a barcode reader to overcome the queue at billing counter. Through their research, they have found that introducing NFC reader to the trolley can maintain the total of the items purchased. They propose introducing IoT interface to all trolleys with the focal server and giving arrangement of online installment would add greater inspiration to shopping knowledge proposed as future work.

\subsection{Technology Review}

Developing object recognition Venkateswarlu [7] presents an efficient way of color to greyscale conversion. Image representation is the primary stage in image processing. Image can be represented in RGB, CMY, YUV and HSV. Then the image is compressed since the larger images costs more in terms of memory by using Lossless and Lossy techniques. As the next step image is enhanced. Image enhancement falls into two categories as spatial domain and frequency domain, transformation based and histogram. Image enhancement techniques include the noise reduction by using linear smoothing, Median filters, changing the brightness which increases the smoothness of the image, histogram equalization and de-blurring by smoothing filters. Image is analyzed by edge detection and segmentation. Final stage is the image understanding which includes the techniques optical character recognition, pattern recognition, object recognition and object motion tracking. As the first step of color conversion to greyscale image is read by RGB pixel values, then calculate 8-bit grey value and result the pixel as 8-bit grey value.

Pandit and Rangole [8] through presented a review on image processing techniques used in object counting. First step is to capture the image by a quality camera. Then the image is enhanced using gray level transformations, histogram modifications, average and median filters etc. And the resulted image is segmented using data clustering or edge based clustering. Finally, objects are counted from the segmented areas by using blob analysis, connected component analysis, statistical area measurements etc. The sources elements in object counting are quantitative analysis, motion tracking and qualitative analysis. They suggest using these techniques in quality control and industrial packaging systems with a quality camera.

Ushma and Shanavas [9] described an efficient way of object detection by image enhancement and edge detection. First the input image is transformed to gray level. Then using morphological operations image is enhanced. There they have used the SURF command and subtract background image, background from the original image which is helpful to detect edges. Since the image is too dark after removing background they have used imadjust to adjust the contrast of the image and edge detection algorithm is applied. Canny's edge detection algorithm is used since it yields good results in detecting horizontal and vertical edges and circular edges and edges at the corner same time.

Latharani, Kurian and Murthy [10] through their paper present few object recognition techniques in computer vision and challenges when using these techniques. They have mentioned reliability, speed and automation are the important factors to be considered when choosing an algorithm for object recognition. They have shown that appearance based object recognition is the best algorithm for 3D object recognition as this method covers the whole image by global features and a single point or smaller area by local features. But clutter, occlusion, background changes and usage of simple conjunctions of low level features limit this algorithm.

According to them, for an industrial environment model based approach is the best as its objects are known before or previously defined. Important feature of this method is division of matching process into preprocessing units which reduces the complexity of the algorithm and provide a straightforward approach. Template based object recognition is best to find smaller areas in an image. Part based object recognition is used in generic recognition problems as it provides qualitative description of visual appearance. There are two stages in contour based object recognition as locate the object and describe features, then matches the shapes to prototypes. However, comparing and querying images with colors and texture by using these algorithms are challenging.

Khurana and Awasthi [11] discussed about the various techniques use in identifying multi objects in an image. They have shown that the template matching requires vast database of picture formats for accurate object

recognition. Thus, it must be utilized just when restricted articles are to be distinguished. Global features and shape based strategy can give better outcome and are proficient when contrasted with nearby elements. These procedures help in simple access of the pictures. They likewise discover their application in fields, for example, biometric recognition, medicinal examination, surveillance, and so forth. A technique for numerous protest identification is additionally exhibited. The strategy trains distinctive question identifiers with individual items. There are $\mathrm{N}$ protest identifiers which are prepared to recognize $\mathrm{N}$ distinctive articles

Any of the previously mentioned object recognition methods can be utilized relying on the application region. A picture is given as contribution to the framework. A similar picture is given as contribution to all protest identifiers. Every identifier will figure out whether the object is available or not. They propose to utilize object finder alongside limit locator. If the protest is available, the identifier will discover its limit and tag the object name in the picture. In this way, after the picture has passed by means of the considerable number of indicators all articles will be recognized alongside object limit and its tag showed in the yield picture. 
Additionally, when the yield picture is shown and can move the cursor over the picture. The tag appeared for a protest inside the total limit of the object stays same. Such multiobjects identification in the picture can extraordinarily enhance the execution of the substance based picture recovery frameworks. The execution can further be enhanced by giving the object locators a chance to keep running in parallel.

Biswas and Hossain [12] through their work presented an automated vegetable recognition system. First, they take pictures of vegetables and classify them according to color, shape, size, weight and texture. They captured take lights-off image and lights-on image when capturing the input image and extract foreground image from background. Then they made histogram of color features, texture features, shape features, size features and concatenated them to compare and contrast with the histograms stored in the database. To remove noise and blur effects they have used HSV technique. To detect children of a parent of vegetable texture classification was used with gray level threshold. They propose to develop the system by quantifying and qualifying with vegetables like cucumbers, tomatoes, mushrooms, potatoes etc.

Muhtaseb, Sarahneh and Tamimi [13] developed an application to distinguish fruits and vegetables based on color and size. First, they input an image of a vegetable or fruit. Then they convert it into HSV model to detect objects since the HSV model is not sensitive for illumination and calculate the histogram of the image. As the second step, they capture an image of intended fruit or vegetable to be identified and follow the same procedure as above. Finally, they compare histograms in two processes and recognize the fruit or vegetable. For the comparison of histogram, they use Chi=square method as it considers the minimum distance classifier. This system shows $75 \%$ accuracy and they suggest enhancing the system with a user-friendly interface and expanding the range of items which increase the performance of the system.

Vegetable Vision [14] is a system which identify vegetables and fruits in supermarkets by their color, shape, size, texture and shape. First, they read the image in MATLAB. They have stored a reference image to compare the original image. Then they convert the image to a black and white image using im2bw. They used morphological function Strel() which creates a flat disk shaped structuring element. And isolate the area of interest in the image by a bounding box. Feature extraction 'regionprops' is used to extract features and locate the area. Finally, the pixel value is calculated using impixel inbuilt command and imcrop to crop the image in MATLAB and find the centroid. Using this algorithm, they have identified tomato, chilly, brinjal, potato, peas, bitter gourd, pumpkin, carrot, bronchi, maize and capsicum. The writer motivates to apply the proposed algorithm in the areas like surgery planning, face recognition, locate tumors and other pathologies, measure tissue volumes and many more.

Senthamaraikannan, Shriram and William [15] through their work real time color recognition on the colors red, blue and green. The experiment was done on color based image segmentation, non-stationary color based target tracking, color based mouse pointer, color based virtual music instruments and color based virtual calculator. The input image is subjected to number of frame images. First it is converted to gray and RGB values are subtracted from the gray image. Then the resulted image is converted to a binary image. Pixel values are multiplied between two or more images. Finally, the colors are recognized. The writer proposes to implement a robot for spying purpose with the introduced algorithm which helps for color recognition.

Buksh, Routh, Mitra, Banik, Malik and Gupta [16] in their paper describes about the using of different MATLAB functions available in image processing tool box of MATLAB and utilizing the same to make an essential image processor having distinctive elements like, review the red, green and blue segments of a shading picture independently, shading recognition and different components like noise removal and addition, edge location, trimming, resizing, revolution, histogram modify, brightness control etc. that is utilized as a part of a fundamental image processing editor along object identification and following. They suggest expanding the set of applications as the future work.

Navada and Santhosh [17] presents an effective prototype for color blind people in detecting color and edges of a given image. First image is captured by a wireless camera and the input image is digitized using TV tuner card. The resulted image is processed using LABVIEW to find color and edges of the image. To obtain information of the image from video edge detection was used as it reduces the amount of data to be processed. Using dialation and erosion morphological features noises are removed. Since both edges and noises present high frequency, noises should be removed. Finally smoothing, enhancement, detection and localization is applied respectively. Smoothing reduces more noises without destroying true edges. Through enhancement the quality of the edges is sharpen. Edge thinning and linking is done in localizing. They hope to extend the system by converting the recognized text to speech and sending to blind people as the future work.

Moghimi [18] proposes an efficient way for classification using colors. First, he extracted local and global features of the image using histograms. Marginal histograms of different color channels of a set of color spaces are used to extract color features. $\mathrm{K}$ nearest method is used to classify the data set. They used this algorithm to identify birds in a particular area. Dealing with confusion matrices and color consistency are the problems he undergoes in his work. Writer propose to use Multiple Kernel Learning to build a color based classifier using color feature as the future work.

Hirano, Garcia, Sukthankar and Hoogs [19] via their paper present the applications of computer vision in the industry. First, they discussed about the Visual Recognition at France Telecom Research and Development. There they have overcome the challenge "Pascal Visual Object Class Challenge" for object recognition by training a labelled set of images and various test of images to achieve the conclusion of supervised algorithms trained with reduced and trained set of models. At Intel for visual recognition they have used sub image using local descriptors to extract same features with query images in a database, object based image retrieval to identify objects from target based user provided set of objects from online and computational Nano-vision to overcome the challenge by the low resolution and the low signal-to-noise ratio of Nano imaging tools. General Electric Global Research presents scene content classification to label every pixel in an image with its category, person re-identification using Spatio-temporal appearance. At Toyota used Adaboost algorithm to overcome the challenge to identify pedestrians 
in large appearance, large self of occlusions and overlap between people.

Wilson [20] in his paper presents an algorithm for real time multiple objects tracking using RGB color space in video. Preprocessing and thresholding is used to color conversion and identify all the objects in the video respectively. To calculate the threshold value of each color RGB color space was used. For the color discrimination, linear model was used. Then the morphological operation imfill is applied. Blob analysis is used to identify the required object in binary image. The writer motivates to use the same techniques to other colors as the future works.

\section{DESIGN AND IMPLEMENTATION OF THE SOLUTION \\ 3.1 The high level architecture of the system}

Architectural design represents the overall structure of the Smart veggie identification system for supermarkets, which develops to manage and maintain the information in several functions. Main activity of the users is to fill the baskets quick as possible when they are notified. The solution is developed as it meets the minimum requirements to develop a web application. As the figure one shows it consists of three layers, Presentation, Logic and Data Layer where the logic layer has an intelligent business layer which is considered the core of the platform.

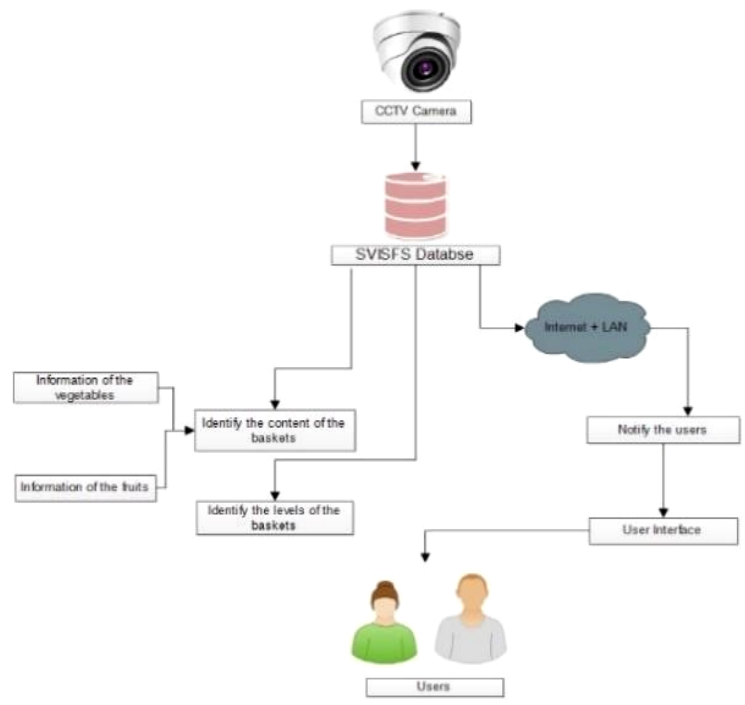

Figure 1: Software Architecture

\subsection{Design of the System Core}

The system is design to identify the contents of the baskets which contains vegetables and fruits. The first task is to extract a single item from the image taken of the basket and identify that. After extracting the item using image slicing methods, the system is developed to identify the relevant vegetables and fruits. As the initial step, Scale-Invariant Feature Transform (SIFT) method in image processing to detect items was used. However, it did not yield the expected results as it was directing to some other items as well.

Template matching was the next step to extract features of vegetables and fruits. It is not an efficient way to identify vegetables though it was able to extract some of the features. Afterwards Speeded-Up Robust Features (SURF) method was used to identify the vegetables and fruits. Though it is much faster than SIFT method results were not accurate as expected. Then thresholding method was used. It gave accurate results, but it could not identify the vegetable separately.

After trying above methods, neural networks gave many accurate and efficient results and used the image slicing method to develop the system further. Neural network with seven layers was used to train two vegetables eggplant and tomato and two fruits, apple and orange. The neural network gave $80 \%$ accuracy. Below are some of the test results obtained and system has been tested for two vegetables and two fruits.

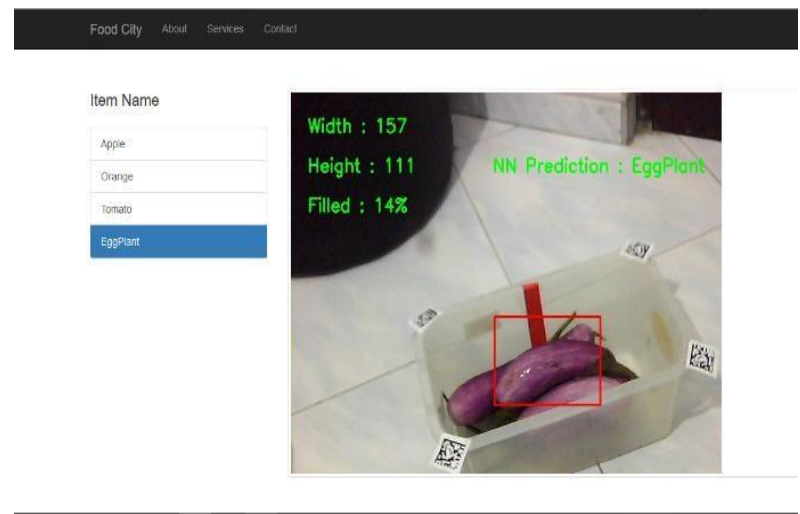

Figure 2: Final test results for eggplant

\section{HOW SYSTEM WORKS}

The proposed system can identify the content of the basket by analyzing an image taken by the CCTV camera system. The system is developed to identify fruits and vegetables only. The system has been developed using image processing techniques and neural networks. To detect the different levels in the baskets, a color strip had been used. From this color strip, the filled amount is calculated. For each basket there is a specific color strip with a known height as you can see in the figure 2. This indicates the level of the content filled in the basket.

A Neural network was trained to identify the content of the basket. From four virtual markers on the top of the basket, an image is captured and cropped. This image is fed into the neural network as the input for the identification of content of the basket. One can claim that we can use pressure sensors for the baskets without using image processing. However, it should be highlighted that the method that presented is much cost effective than wiring all the baskets and spending money on sensors. And also, the system has implemented from the existing CCTV cameras that are already fixed at supermarkets and the owners do not want to spend extra money on pressure sensors. The system also provided with the specific location of the basket which is nearly to be emptied.

\section{CONCLUSION AND FUTURE WORKS}

The implemented system consists with mainly three modules. One is the module to identify the level of the basket content. For this concept, color classification method has been used and small calculation was done with the height of the stripe. Second module is to identify the level of the basket. Neural network has been used to identify the fruits and vegetables stored in the baskets. Third is to notify the responsible parties and system was integrated with Raspberry pi chip set.

Implemented project is a system to identify user preferences when buying commodities and provide efficient service to 
the customers with the use of image processing and neural networks and give the users the best displaying option depending on their appetite and need, and also optimize the available resources. The neural network yields an accuracy of $80.33 \%$ when identifying content of the basket. The system is tested with two vegetables egg plants and tomatoes as well as two fruits apple and oranges. Egg plants and apple had shown good accuracy in result, while the accuracy of the results for tomatoes and oranges are average. In future the system can be developed and tested for more accurate results when identifying vegetables and fruits. Furthermore, nature inspired algorithms such as ant colony optimization can be implemented to detect edges in the extract images of the vegetables and fruits when identifying those items. This concept can be extended to identify other items in supermarkets as well.

\section{REFERENCES}

[1] S. Sojitra and G. Patel, "A Review of Smart Shopping Systems," 2016.

[2] R. M. Bolle, J. H. Connell, N. Haas, R. Mohan, and G. Taubin, "VeggieVision: a produce recognition system," 1996, pp. 244-251.

[3] W.

Applebaum "studying customerbehaviorinretailstores-090623023217phpapp01.”

[4] C. M. Sukanya, R. Gokul, and V. Paul, "A Survey on Object Recognition Methods," Int. J. Sci. Eng. Comput. Technol., vol. 6, no. 1, p. 48, 2016.

[5] D. Lu, D. A. Kiewit, and J. Zhang, Market research method and system for collecting retail store and shopper market research data. Google Patents, 1994.

[6] Z. Ali, "RFID Based Smart Shopping and Billing," 2013.

[7] I. B. Venkateswarlu, "Analytical Survey of Colour to Greyscale Conversion Methods Based on Primary Image Processing Techniques.” International Journal of Advanced Research in Computer Science and Software Engineering, 10-Oct-2014.

[8] A. Pandit and J. Rangole, "Literature Review On Object Counting Using Image Processing Techniques." International Journal of Advanced Research in Electrical, Electronics and Instrumentation Engineering, 04-Apr-2014.
[9] A. Ushma and P. D. A. R. M. Shanavas, "Object Detection In Image Processing Using Edge Detection Techniques.” IOSR Journal of Engineering (IOSRJEN), Mar-2014.

[10] International Conference on Pattern Recognition, A. Sanfeliu, and International Association for Pattern Recognition, Eds., VARIOUS OBJECT RECOGNITION TECHNIQUES FOR COMPUTER VISION. Los Alamitos, Calif.: IEEE Computer Society, 2000 .

[11] K. Khurana and R. Awasthi, "Techniques for object recognition in images and multi-object detection," Int. J. Adv. Res. Comput. Eng. Technol. IJARCET, vol. 2, no. 4, pp. 1383-1388, 2013.

[12] L. G. Shapiro and G. C. Stockman, Computer vision. Upper Saddle River, NJ: Prentice Hall, 2001.

[13] A. Muhtaseb, S. Sarahneh, and H. Tamimi, "FruitVegetable_Recognition." ReearchGate, 12-Feb2015.

[14] "Image_Segmentation_and_Recognition_Using.".

[15] D. Senthamaraikannan, S. Shriram, and J. William, "Real time color recognition," Int. J. Innov. Res. Electr. Electron. Instrum. Control Eng., vol. 2, no. 3, 2014.

[16] R. Buksh, S. Routh, P. Mitra, S. Banik, A. Mallik, and S. D. Gupta, "MATLAB based image editing and color detection," Int. J. Sci. Res. Publ., vol. 4, no. 1, pp. 1-6, 2014

[17] B. R. Navada, K. V. Santhosh, S. Prajwal, and H. B. Shetty, "An image processing technique for color detection and distinguish patterns with similar color: An aid for color blind people," 2014, pp. 333-336.

[18] M. Moghimi, "Using color for object recognition," Calif. Inst. Technol. Tech Rep, 2011.

[19] Y. Hirano, C. Garcia, R. Sukthankar, and A. Hoogs, "Industry and object recognition: Applications, applied research and challenges," in Toward Category-Level Object Recognition, Springer, 2006, pp. 49-64.

[20] K. Wilson, "Real- Time Tracking for Multiple Objects Based on Implementation of RGB Color Space in Video," Int. J. Signal Process. Image Process. Pattern Recognit., vol. 9, no. 4, pp. 331-338, Apr. 2016 